\title{
Gammagrafía de ventilación/perfusión pulmonar: utilidad en el estudio de hipertensión pulmonar y embolismo pulmonar crónico
}

\author{
Ventilation / perfusion scan Pulmonary: \\ utility in the study of hypertension pulmonary \\ and chronic pulmonary embolism
}

\author{
Víctor Marín Oyaga, MD, MSc ${ }^{1,2}$ Rafael Conde Camacho, MD ${ }^{3}$ Claudia GutiérRez Villamil, MD ${ }^{1,2}$ \\ CAmila Vélez GutiérReZ, MD² Sinay Arévalo Leal, MSc, PhD. ${ }^{1,2}$
}

\section{Resumen}

La hipertensión pulmonar tromboembólica crónica (HPTC) es una enfermedad grave con una importante morbimortalidad asociada. Las imágenes diagnósticas juegan un papel fundamental tanto en el diagnóstico como en la determinación de extensión, distribución y gravedad, para así establecer la posibilidad del manejo quirúrgico.

La gammagrafía de ventilación/perfusión (G V/Q) tiene una alta sensibilidad y valor predictivo negativo, permite descartar la presencia de tromboembolismo pulmonar (TEP) como una causa de hipertensión pulmonar y está indicada en el estudio inicial de estos pacientes.

Debido a su importancia resaltada recientemente en diferentes guías nacionales e internacionales de manejo, es importante familiarizar a los médicos tratantes con la adecuada metodología de realización, así como con el enfoque en su interpretación debido a que es diferente cuando se trata de estudios para TEP agudo. Se presentan dos casos clínicos en los que el estudio fue fundamental para el diagnóstico y tratamiento oportuno de HPTC con evolución favorable.

Palabras clave: cintigrafía, imagen de perfusión, ventilación pulmonar, embolia pulmonar. 


\section{Introducción}

La hipertensión pulmonar tromboembólica crónica (HPTC) se define como el incremento de presión en la arteria pulmonar con una media en reposo mayor o igual a $25 \mathrm{~mm} \mathrm{Hg}$, en el contexto de un paciente con antecedente de tromboembolismo pulmonar (TEP), quien recibió manejo anticoagulante efectivo por al menos tres meses (1).

El TEP tiene una incidencia de 112 casos por cada 100000 habitantes (2), de los cuales se estima que el 1 $\%-3,8 \%$ de los pacientes presenta HPTC. No hay datos exactos sobre su incidencia; sin embargo, se ha estimado una incidencia acumulada del $1 \%$ (a 6 meses) y 3,8 $\%$ (a 3 años) en pacientes con un episodio previo de TEP, en quienes se hizo un seguimiento cercano (3-5). Se ha reportado que hasta en un $25 \%$ de los pacientes con HPTC no se documenta un episodio previo de TEP agudo, e incluso hasta el $44 \%$ no tenía antecedentes de trombosis venosa profunda (6).

Después de un episodio de TEP agudo, aproximadamente el $70 \%$-85\% de los pacientes presenta resolución completa del trombo; sin embargo, aquellos pacientes con resolución parcial presentan cambios inflamatorios crónicos que afectan el lumen de los vasos pulmonares, por un mecanismo aún poco conocido, finalmente llevando a una recanalización parcial del flujo sanguíneo (1). Usualmente, estos pacientes se presentan con síntomas inespecíficos como disnea y deterioro en la clase funcional, dolor torácico, síncope o hemoptisis (5).

La HTPC es una enfermedad grave con una importante morbimortalidad asociada. Para poderla diferenciar de la enfermedad subaguda, deben haber transcurrido tres meses de anticoagulación efectiva. Se estima que la HTPC es una de las formas más graves de hipertensión pulmonar, aunque es la única que puede tener un tratamiento efectivo. Está clasificada como grupo 4 en las guías de hipertensión pulmonar (HTP) de la Sociedad Europea de Cardiología (ESC)/European Respiratory Society (ERS) del 2015 (7).
La importancia de realizar un diagnóstico adecuado yace en la posibilidad de un tratamiento oportuno por diferentes métodos. Es fundamental hacer un diagnóstico temprano de esta entidad, pues el retraso en el diagnóstico tiene un fuerte impacto en el pronóstico del paciente. Se estima que la supervivencia de pacientes con presión media de la arteria pulmonar mayor a $50 \mathrm{~mm} \mathrm{Hg}$ al momento del diagnóstico es menor del $20 \%$ a 2 años. Sin embargo, aumenta a un $80 \%$ a 3 años una vez se realiza el procedimiento quirúrgico de endarterectomía de forma exitosa (8).

Las imágenes diagnósticas juegan un papel fundamental tanto en el diagnóstico como en la determinación de la extensión, distribución y gravedad, para así establecer la posibilidad del manejo quirúrgico (6). Uno de los principales métodos diagnósticos es la gammagrafía de ventilación/perfusión pulmonar $(\mathrm{G}$ V/Q), debido a su alta sensibilidad para detectar el TEP crónico como una causa de HTP.

Se presentan dos casos en los que el estudio G V/Q fue fundamental para el diagnóstico y tratamiento oportunos, con evolución clínica adecuada posterior al tratamiento.

\section{Caso clínico 1}

Paciente mujer de 56 años en estudio por disnea y cor pulmonale con HTP documentada. Dentro del estudio etiológico se solicitó una angioTAC de vasos pulmonares que no mostró hallazgos compatibles con TEP. Ante la continuidad de la sospecha clínica de HPTC se solicitó una G V/Q que mostró defectos de tipo vascular de perfusión de predominio periférico, la mayoría de ellos sin pérdida del borde pulmonar sin que ninguno de ellos fuese concordante con defectos de ventilación, por lo que el estudio se interpretó como compatible con TEP crónico (Figura 1).

Ante dichos hallazgos, se llevó a angiografía pulmonar en la que se identificaron lesiones obstructivas en las ramas de la arteria pulmonar izquierda, por lo que se decidió llevar a angioplastia con balón, con un desenlace angiográfico y clínico favorable. 


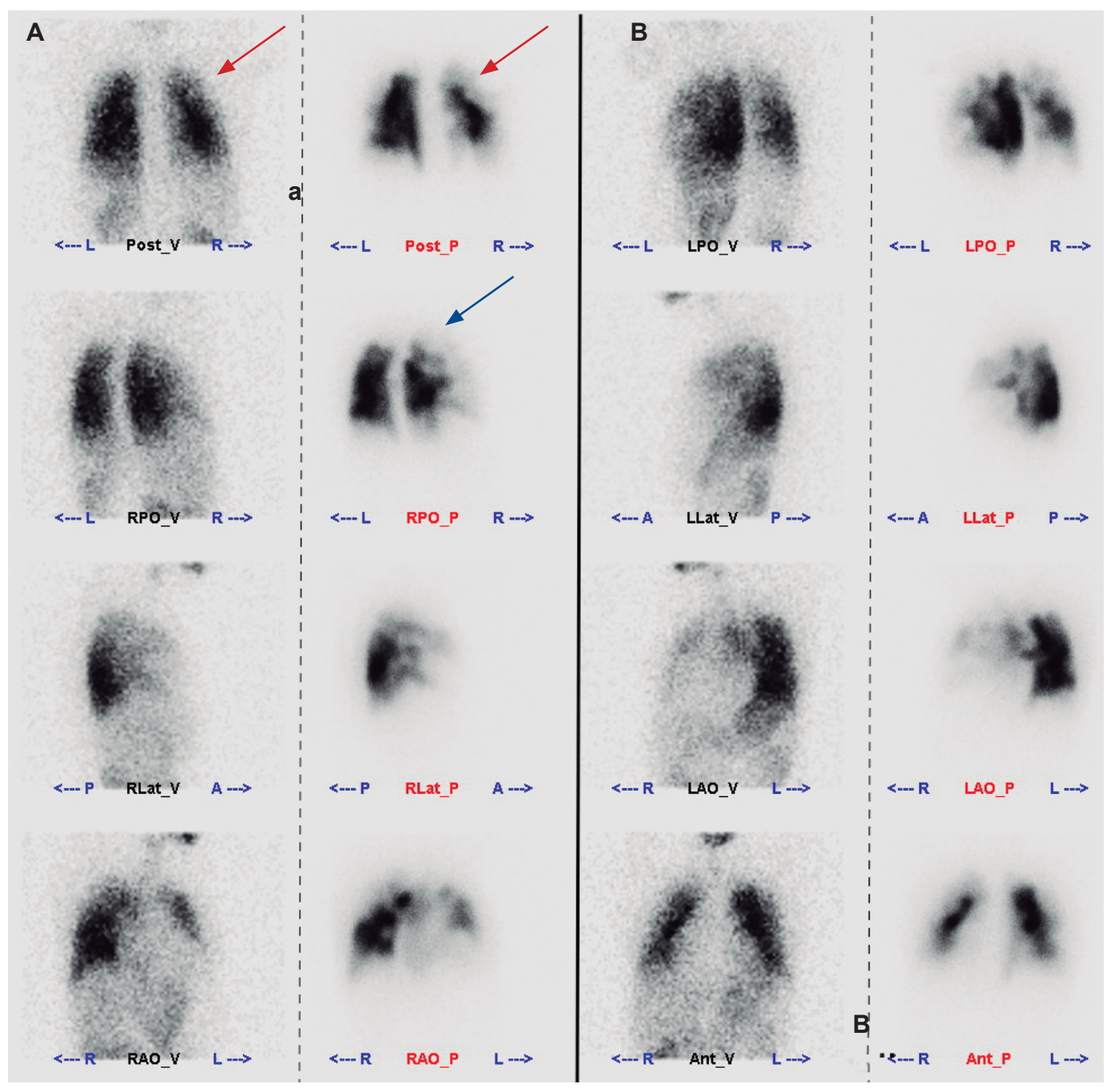

Figura 1. Estudio gammagráfico de ventilación (columnas a la izquierda de la línea discontinua) y perfusión (columnas a la derecha de la línea discontinua). Se realizaron imágenes planares en proyecciones oblicuas anteriores, oblicuas posteriores y laterales del pulmón derecho (A) e izquierdo (B), así como imágenes en proyección anterior y posterior ( $\mathbf{a}$ y b). En el estudio de perfusión, la distribución del radiotrazador se realiza de manera heterogénea en ambos pulmones debido a múltiples defectos de predominio periférico, algunos de ellos con un patrón de rueda dentada (flechas azules) que se consideran de tipo vascular. Muchos de ellos no presentan pérdida total del borde pulmonar. La silueta cardíaca está aumentada de tamaño a expensas de las cavidades derechas. En el estudio de la ventilación se observa una concentración adecuada y homogénea en ambos campos pulmonares sin retenciones groseras o evidentes en las vías aéreas, y todas las zonas con defecto de perfusión son discordantes con el estudio de ventilación -miss-match- (flechas rojas) compatible con TEP bilateral.

\section{Caso clínico 2}

Paciente hombre de 72 años con antecedente de TEP antiguo hace aproximadamente tres años en anticoagulación crónica. También tenía historia de HTP grave con presión sistólica en la arteria pulmonar de 89 mm Hg. Requiere un suplemento de oxígeno. Consultó por un cuadro de un día de disnea súbita y dolor torácico de tipo pleurítico en el tórax izquierdo sin otros síntomas respiratorios asociados. Se le realizó angioTAC del tórax para el estudio de TEP que mostró un defecto que afecta la pared medial de la arteria interlobar 
derecha con extensión a la arteria segmentaria basal medial del lóbulo inferior ipsilateral compatible con un trombo adherido recanalizado que afecta el $50 \%$ de la luz del vaso, así como atelectasias en el lóbulo inferior del pulmón izquierdo. Se realizó un estudio de G V/Q con hallazgos de defectos parciales de perfusión y de tipo vascular en su mayoría sin compromiso del borde pulmonar en ambos pulmones con ventilación preservada (hallazgos no concordantes), por lo que se consideró como un estudio anormal compatible con TEP crónico-antiguo con recanalización parcial y bilateral (como hallazgo adicional a la angioTAC) (Figura 2).
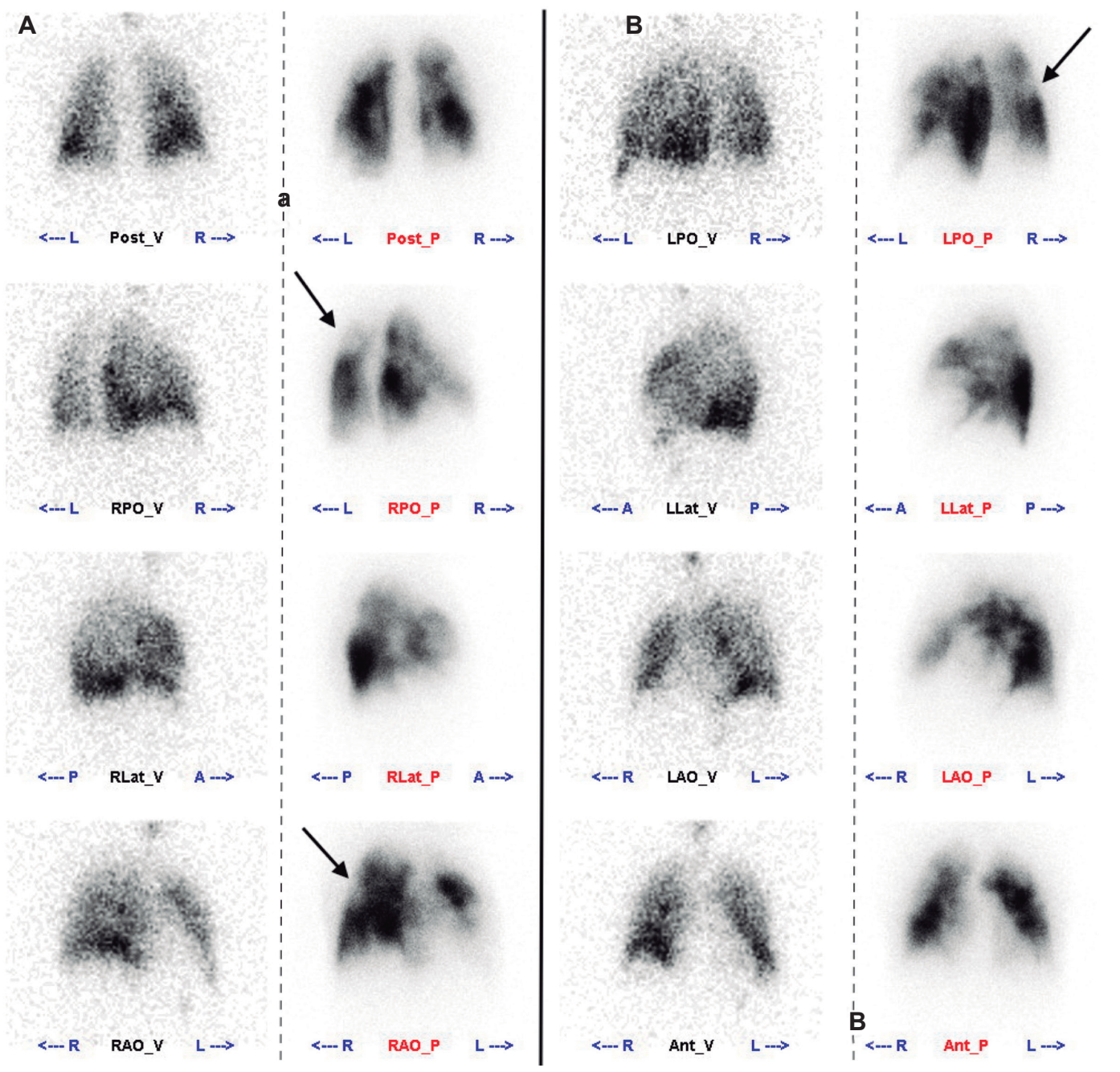

\&-L LPO Y R
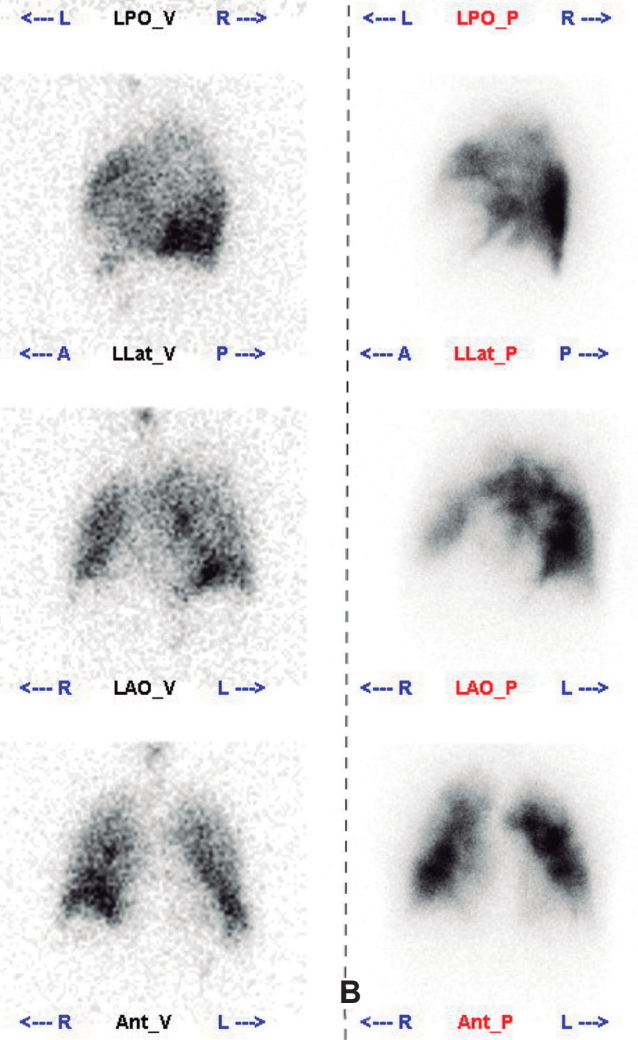

Figura 2. Estudio gammagráfico de ventilación (columnas a la izquierda de la línea discontinua) y perfusión (columnas a la derecha de la línea discontinua). Se realizaron imágenes planares de en proyecciones oblicuas anteriores, oblicuas posteriores y laterales del pulmón derecho (A) e izquierdo (B), así como imágenes en proyección anterior y posterior (a y b). La distribución precapilar pulmonar de las radiopartículas de albúmina es anormal debido a la presencia de múltiples defectos de perfusión que no tienen correlato aéreo. Los defectos de perfusión son de predominio periférico y mixtos con y sin pérdida del borde pulmonar (flechas negras). La ventilación a nivel alveolar pulmonar bilateral es prácticamente normal. Por tanto, se observan defectos no concordantes entre la perfusión y la ventilación, y de tipo mixto compatible con TEP probablemente crónico. 
Con dichos hallazgos se llevó a cateterismo cardíaco derecho y angiografía pulmonar con signos compatibles con TEP bilateral; posteriormente, se programó para endarterectomía bilateral, en la cual se hallaron trombos arteriales bilaterales que se extrajeron con éxito, con posterior mejoría clínica, de clase funcional; y finalizó sin requerimientos de oxígeno.

\section{Discusión}

En los dos casos presentados se muestra la importancia de la $\mathrm{G}$ V/Q dentro del algoritmo diagnóstico de la HTP por TEP crónico y se demuestra que es un examen complementario e incluso superior para el enfoque del paciente con esta enfermedad, en comparación con la angioTAC.

Las más recientes guías europeas de diagnóstico y tratamiento para la HTP proponen a la G V/Q como herramienta recomendada para descartar o para tener como posibilidad diagnóstica la HPTC (Figura 3). Todo lo anterior debido a su alta sensibilidad en el estudio de TEP crónico en comparación con la angioTAC. Una prueba $\mathrm{G} V / \mathrm{Q}$ negativa prácticamente descarta la enfermedad tromboembólica crónica (9).

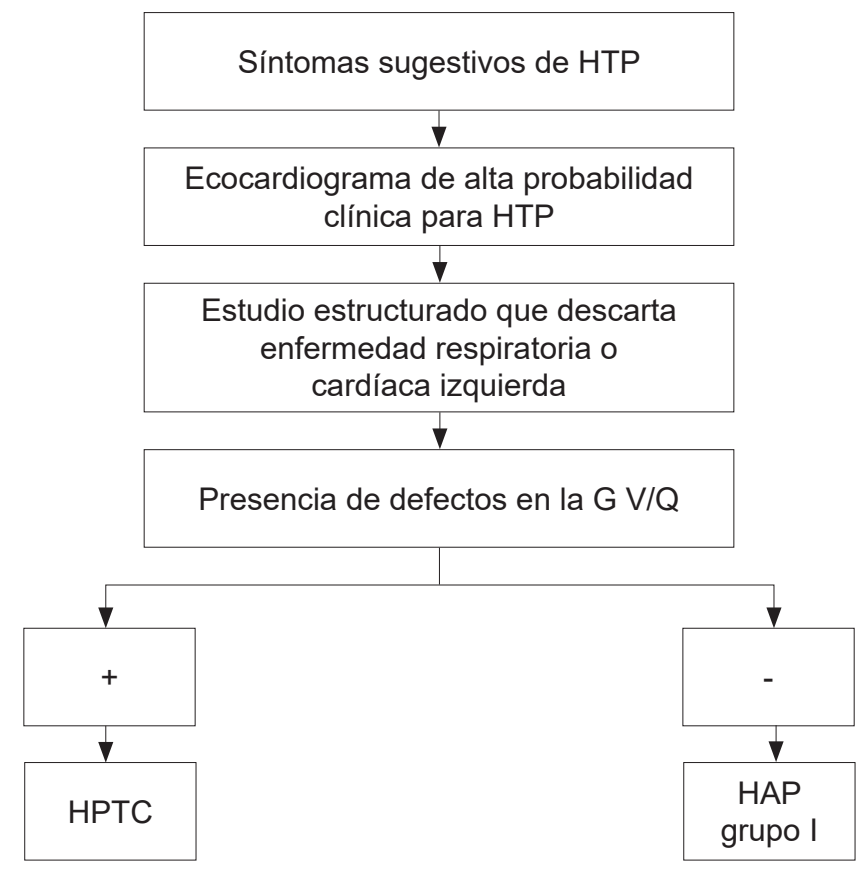

Figura 3. Algoritmo diagnóstico en la hipertensión arterial pulmonar (HAP). Modificado de: Galiè N. Rev Esp Cardiol. 2016;69(2):102-810.
El diagnóstico del embolismo pulmonar crónico en el contexto de HTP y el descarte de posibles diagnósticos diferenciales es fundamental en la actualidad debido al impacto sobre un posible tratamiento curativo para la enfermedad como la endarterectomía pulmonar (9).

\section{G V/Q técnica e interpretación}

La prueba completa consta de dos fases: la de perfusión y la de ventilación. En algunas ocasiones específicas y predominantemente cuando la sospecha clínica es por TEP agudo se puede realizar solo la fase de perfusión comparándola con alguna imagen radiológica previa. La fase de perfusión se realiza después de la administración endovenosa de macroagregados de albúmina marcados con ${ }^{99} \mathrm{~m}$-Tc (99mTc-MAA). El fundamento principal es que, debido al tamaño de las partículas (15$100 \mu \mathrm{m}$ ), estas seguirán la circulación pulmonar normal y se alojarán en los capilares pulmonares distales o en áreas de los defectos secundarios al embolismo crónico. La dosis para administrar recomendada en adultos es en promedio de 1-6 mCi (37-222 MBq) endovenosos.

La fase de ventilación se realiza con gases inertes o con aerosoles radiomarcados como el ${ }^{99} \mathrm{mTc}$-DTPA, una fracción del material aspirado se aloja en los alveolos pulmonares siguiendo el patrón de ventilación normal del paciente o mostrando los defectos secundarios a la patología parenquimatosa previa.

La G V/Q se puede realizar con la obtención de imágenes planares (dos dimensiones) o con imágenes tomográficas tipo SPECT (single photon emission tomography) teniendo este último un mayor rendimiento. Usualmente, los defectos de perfusión, debido a su tamaño, se pueden detectar por medio de imágenes planares, a pesar de que estas aportan una menor resolución con respecto a las imágenes obtenidas por SPECT, el cual permite una mayor detección de defectos subsegmentarios (10). Sin embargo, el mayor requerimiento de tiempo del paciente en cámara, la necesidad de quietud del individuo durante la adquisición y la escasa diferencia de rendimiento hacen que el SPECT de perfusión/ ventilación pulmonar no se realice en los centros de medicina nuclear de manera rutinaria.

Debido a la forma esquemáticamente cónica o triangular de los segmentos pulmonares (con su vértice

Revista Colombiana de Neumología Vol. 30 N. ${ }^{\circ} 2 \mid 2018$ 
dirigido hacia el hilio y la base hacia la periferia) y a que la circulación pulmonar no cuenta con colaterales, la presencia de una oclusión arterial clásicamente se ha descrito como ausencia o disminución de la captación del radio trazador en el área hipoperfundida, con una forma triangular de base externa y pérdida del borde externo pulmonar (10). En el transcurso de la historia de la prueba se han utilizado varios criterios de interpretación que básicamente consisten en correlacionar el estudio de perfusión con defectos o no en el estudio ventilatorio o su correlación con hallazgos radiológicos que expliquen dicho defecto. Clásicamente se considera un estudio compatible con TEP aquel en el que se observen hallazgos o defectos no concordantes o no coincidentes entre el estudio de perfusión y de ventilación (11).

Estos criterios de interpretación también aplican para el estudio de TEP crónico. Sin embargo, se ha observado que dicho patrón en esta entidad no es tan clásico, y los defectos de perfusión de cualquier intensidad (sin que sean ausencias puras de perfusión e incluso con preservación del borde pulmonar) y de distribución mucho más irregulares y difusos que presenten algún grado de mejor ventilación también pueden ser sugestivos de TEP crónico. Lo anterior se debe a la embolia pulmonar crónica con recanalización y localización en diferentes territorios (12). En el TEP crónico, al haber una recanalización parcial del trombo, existe la posibilidad de paso de algunas partículas de macroagregados radiomarcados, lo que se traduce en una imagen con menor captación del radiotrazador, sin evidenciarse el patrón clásico previamente descrito, por lo que es posible que se subestime la gravedad de la obstrucción vascular. También puede mostrar pequeños defectos periféricos múltiples (patrón de rueda dentada) o únicos, así como defectos no segmentarios en la perfusión que no se observan en la ventilación.

Una $G$ V/Q normal y sin defectos no concordantes prácticamente excluye el diagnóstico de TEP crónico con una sensibilidad del $90 \%-100 \%$ y una especificidad del $94 \%-100 \%(6,7)$. Esta prueba no tiene contraindicaciones especiales y tan solo se deben tener algunos cuidados como la reducción de cantidad de partículas en algunos pacientes con HTP grave.
La angioTAC es otro método ampliamente utilizado en el diagnóstico de TEP. Permite realizar una reconstrucción en tres dimensiones del árbol vascular con una alta resolución $(0,5 \mathrm{~mm})$ y visualizar el parénquima pulmonar y el mediastino. Tiene una alta sensibilidad en el diagnóstico de TEP agudo (el 97 \%-98,3 $\%$ a nivel lobar y $92,9 \%-94,6 \%$ a nivel segmentario). Sin embargo, su utilidad se limita de forma significativa en el diagnóstico de TEP crónico (13). Tunariu y colaboradores realizaron un estudio retrospectivo en el cual compararon la G V/Q con la angioTAC en 227 pacientes con HTP. Demostraron una mayor sensibilidad (96\%-97\%) y especificidad (90\%-95\%) de la G V/Q en la detección de HPTC, con respecto a la angioTAC (con una sensibilidad del $51 \%$ y especificidad del $99 \%$ ). De este modo, una G V/Q normal permite descartar la presencia de HPTC a diferencia de una angioTAC normal. La angioTAC con contraste es útil para determinar si la HPTC es accesible por cirugía, para identificar los hallazgos angiográficos típicos de la HPTC y los colaterales procedentes de las arterias bronquiales; por tanto, se pueden usar de forma complementaria (Figura 4) $(7,14)$.

Existen aún campos por explorar en cuanto al estudio de G V/Q y a las posibilidades de información adicional que podría dar. Como ya se comentó, una de ellas es la posible realización de rutina de estudio SPECT o SPECT/CT en lugar de las proyecciones planares. La discusión está en que, aunque el rendimiento diagnóstico es algo mejor, el mayor tiempo de adquisición y mayor dosis de radiación justifican o no su uso rutinario. Otro campo que valdría la pena explorar (tanto su factibilidad técnica como su relevancia clínica) es el de la posible cuantificación del compromiso tromboembólico, al poder tomar la fracción de los defectos sobre el total del volumen pulmonar o sobre la cantidad de la radiación cuantificada. Igualmente, se plantea la opción para que el estudio G V/Q se considere dentro del seguimiento de los pacientes para valorar la reperfusión después del procedimiento terapéutico.

\section{Conflicto de interés}

Los autores declaran no tener ningún conflicto de interés. 

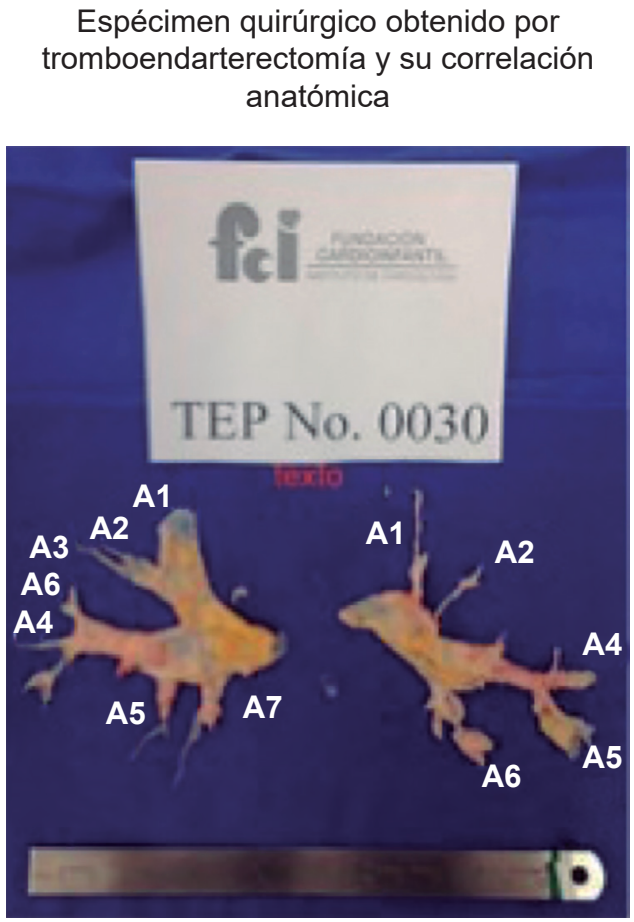

Cortesía Departamento de Cirugía Cardiovascular FCl

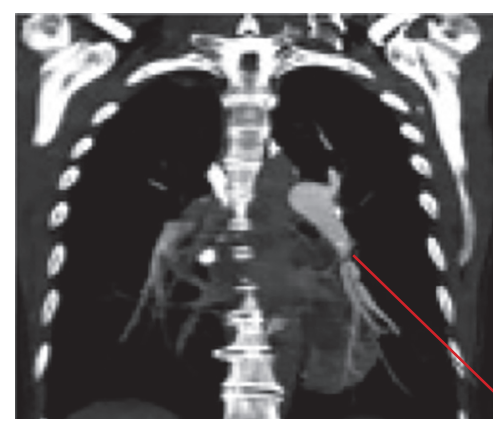

AngioTAC de tórax

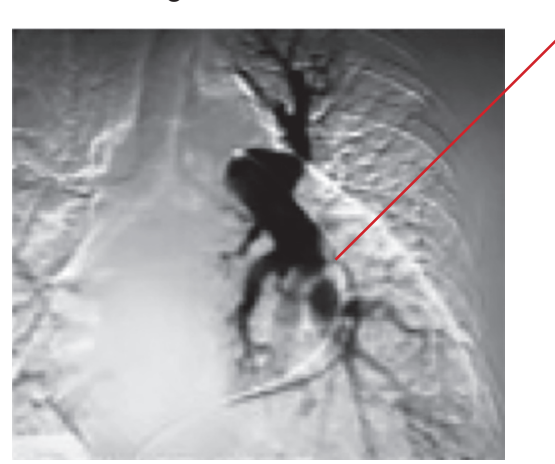

Defectos vasculares

Figura 4. Hallazgos en tromboendarterectomía bilateral y su correlación anatómica con hallazgos en angioTAC y en arteriografía pulmonar. Cortesía del departamento de cirugía cardiovascular. Fundación Cardioinfantil, Instituto de Cardiología.

\section{Referencias}

1. Stricker H. Chronic thromboembolic pulmonary hypertension-a diagnostic and therapeutic update. Vasa. 2016;45(3):195-9.

2. Wiener RS, Schwartz LM, Woloshin S. Time trends in pulmonary embolism in the United States: evidence of overdiagnosis. Arch Intern Med. 2011;171(9):831-7.

3. Pengo V, Lensing AW, Prins MH, Marchiori A, Davidson BL, Tiozzo F, et al. Incidence of chronic thromboembolic pulmonary hypertension after pulmonary embolism. N Engl J Med. 2004;350(22):2257-64.

4. Surie S, Gibson NS, Gerdes VE, Bouma BJ, van Eck-Smit BL, Buller HR, et al. Active search for chronic thromboembolic pulmonary hypertension does not appear indicated after acute pulmonary embolism. Thromb Res. 2010;125(5):e202-5.

5. Tapson VF, Platt DM, Xia F, Teal SA, de la Orden M, Divers $\mathrm{CH}$, et al. Monitoring for Pulmonary Hypertension Following Pulmonary Embolism: The INFORM Study. Am J Med. 2016;129(9):978-985.e2.

6. Gopalan D, Blanchard D, Auger WR. Diagnostic Evaluation of Chronic Thromboembolic Pulmonary Hypertension. Ann Am Thorac Soc. 2016;13 Suppl 3:S222-39.
7. Galiè N. Guía ESC/ERS 2015 sobre diagnóstico y tratamiento de la hipertensión pulmonar. Rev Esp Cardiol. 2016;69(2):102-810.

8. He J, Fang W, Lv B, He JG, Xiong CM, Liu ZH, et al. Diagnosis of chronic thromboembolic pulmonary hypertension: comparison of ventilation/perfusion scanning and multidetector computed tomography pulmonary angiography with pulmonary angiography. Nucl Med Commun. 2012;33(5):459-63.

9. Payares-Jardim C. Diagnóstico y tratamiento de la hipertensión pulmonar tromboembólica crónica. Rev Colomb Cardiol. 2017;24(Suppl 1):48-54.

10. Ruggiero A, Screaton NJ. Imaging of acute and chronic thromboembolic disease: state of the art. Clin Radiol. 2017;72(5):375-88.

11. Bajc M, Neilly JB, Miniati M, Schuemichen C, Meignan M, Jonson B, et al. EANM guidelines for ventilation/perfusion scintigraphy: Part 1. Pulmonary imaging with ventilation/perfusion single photon emission tomography. Eur J Nucl Med Mol Imaging. 2009;36(8):1356-70.

12. Ohira H, Beanlands RS, Davies RA, Mielniczuk L. The role of nuclear imaging in pulmonary hypertension. J Nucl Cardiol. 2015;22(1):141-57. 
13. D’Armini AM. Diagnostic advances and opportunities in chronic thromboembolic pulmonary hypertension. Eur Respir Rev. 2015;24(136):253-62.
14. Tunariu N, Gibbs SJ, Win Z, Gin-Sing W, Graham A, Gishen $\mathrm{P}$, et al. Ventilation-perfusion scintigraphy is more sensitive than multidetector CTPA in detecting chronic thromboembolic pulmonary disease as a treatable cause of pulmonary hypertension. J Nucl Med. 2007;48(5):680-4. 